\title{
Chronic epilepsy associated with temporal tumors: long-term surgical outcome
}

\author{
Dmitry Ruban, M.D., ${ }^{1}$ Richard W. Byrne, M.D., ${ }^{1}$ Andres Kanner, M.D., ${ }^{2}$ \\ Michael Smith, M.D., ${ }^{2}$ Elizabeth J. Cochran, M.D., ${ }^{3}$ David Roh, B.S., 1 \\ and Walter W. Whisler, M.D., Ph.D. ${ }^{1}$ \\ ${ }^{1}$ Departments of Neurosurgery, ${ }^{2}$ Neurology, and ${ }^{3}$ Pathology, Rush University Medical Center, \\ Chicago, Illinois
}

\begin{abstract}
Object. The authors undertook a study to review the clinical features and outcome in patients who underwent surgery for intractable chronic epilepsy caused by temporal lobe tumors.

Methods. The Rush Surgical Epilepsy Database was queried to identify patients with chronic intractable epilepsy who underwent resection of temporal lobe tumors between 1981 and 2005 at Rush University Medical Center. Medical records were reviewed for age of the patient at seizure onset, delay to referral for surgery, seizure frequency and characteristics, preoperative MR imaging results, extent of resection, pathological diagnosis, complications, duration of follow-up period, and seizure improvement.

Results. Thirty-eight patients were identified, all with low-grade tumors. Gangliogliomas were the most common $(36.8 \%)$, followed in descending order by dysembryoplastic neuroepithelial tumors (26.3\%) and low-grade diffuse astrocytoma $(10.5 \%)$. The mean duration between seizure onset and surgery was 15.4 years. Complex partial seizures were the most common presenting symptom. Detailed operative data were available for 28 patients; of these, $89.3 \%$ underwent complete resection of the amygdala, and $82.1 \%$ underwent partial or complete resection of hippocampus, in addition to lesionectomy. The mean follow-up duration was 7.7 years (range 1.0-23.1 years), with $78.9 \%$ of patients having seizure status that improved to Engel Class I, 15.8\% to Engel Class II, and 5.3\% to Engel Class III. Permanent complications were noted in $2.6 \%$ of patients.

Conclusions. The authors' examination of the long-term follow-up data in patients with temporal lobe tumors causing chronic intractable epilepsy demonstrated excellent results in seizure improvement after surgery. (DOI: 10.3171/2009.5.FOCUS0998)
\end{abstract}

Key Words - chronic epilepsy - low-grade glioma - temporal lobe tumor

$\mathrm{S}$ EIZURES are a common manifestation of brain tumors, and chronic medically intractable epilepsy has long been associated with low-grade temporal tumors. ${ }^{1,11,15,16,28}$ The incidence of tumors in patients with chronic epilepsy has been reported to range from 10 to $50 \% .^{1,11,26,27}$ Among these, low-grade gliomas are the most common, with a variety of other histological entities reported. ${ }^{27}$ Although resection is often recommended, management of tumoral temporal lobe epilepsy remains controversial, with some authors advocating lesionectomy alone, and others arguing for more extensive resection. . $^{3,611,17,19,20,29} \mathrm{We}$ present our series of patients with temporal lobe tumors causing intractable chronic epilepsy, focusing on long-term surgical outcomes.

\section{Methods}

The Rush Surgical Epilepsy Database was queried to identify all patients who underwent resection of temporal

Abbreviations used in this paper: DNET = dysembryoplastic neuroepithelial tumor. lobe tumors between 1981 and 2005 at Rush University Medical Center. Only those patients who presented with long-term intractable epilepsy and had long-term followup ( $\geq 1$ year) were included in the study. Medical records were reviewed for age at seizure onset, delay to referral for surgery, seizure frequency and characteristics, preand postoperative MR imaging results, extent of resection, pathological diagnosis, complications, duration of follow-up period, and postsurgical seizure outcome.

\section{Results}

A total of 38 patients with medically intractable epilepsy and pathologically confirmed tumors were identified (Table 1). No patients were excluded from this sample.

The mean age at seizure onset was 12.5 years (range 1-47 years). Complex partial seizures were the most common type, present in 34 patients (89.5\%), followed by generalized tonic-clonic seizures in 11 patients $(28.9 \%)$ and simple partial seizures in 5 patients (13.2\%). Multiple seizure types were documented in 12 patients (31.6\%). 
TABLE 1: Summary of patient demographics

\begin{tabular}{lc}
\hline Parameter & No. of Cases/Value (\%) \\
\hline male & $22(58)$ \\
female & $16(42)$ \\
age at seizure onset (yrs) & \\
$\quad$ mean & 12.5 \\
range & $1-47$ \\
age at op (yrs) & \\
mean & 27.8 \\
$\quad$ range & $6-59$ \\
duration btwn seizure onset \& op (yrs) & \\
$\quad$ mean & 15.4 \\
range & $3-38$ \\
seizure type & \\
$\quad$ complex partial & $34(89.5)$ \\
generalized tonic-clonic & $11(28.9)$ \\
simple partial & $5(13.2)$ \\
seizure frequency/mo & \\
$\quad$ mean & 78.2 \\
range & $2-600$ \\
\hline
\end{tabular}

The mean seizure frequency was 78.2 per month (range $2 /$ month to $20 /$ day).

The mean age at the time of surgery was 27.8 years (range 6-59 years), with mean delay to referral for surgery of 15.4 years (range 3-38 years).

Magnetic resonance imaging records were available for 32 patients (Table 2); in 2 of these patients the imaging findings were reported to be normal, with the tumor discovered incidentally on tissue examination. On T1-weighted sequences, 26 tumors $(86.7 \%)$ were hypointense, $3(10.0 \%)$ were isointense, and 1 (3.3\%) was hyperintense. On T2-weighted sequences, 29 tumors (96.7\%) were hyperintense and 1 (3.3\%) was hypointense. Cystic changes were noted in 5 cases (16.7\%). Typical MR imaging studies are shown in Fig. 1, demonstrating a left temporal lesion that is isointense on T1-weighted imaging and hyperintense on T2-weighted FLAIR imaging in a patient with a ganglioglioma.

Preoperatively, all patients underwent noninvasive video electroencephalography, neuropsychological examination, and Wada testing.
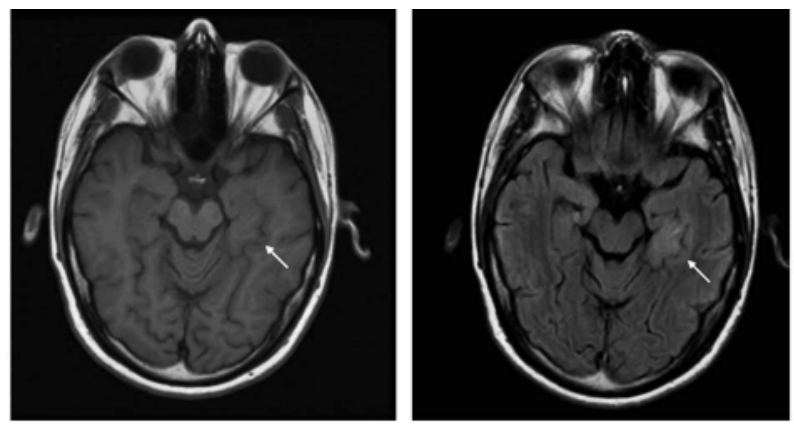

FIG. 1. Representative MR images showing a patient with intractable epilepsy and a left temporal ganglioglioma (arrow).
TABLE 2: Summary of MR imaging characteristics

\begin{tabular}{lc}
\hline Characteristic & No. of Cases (\%) \\
\hline normal & $2(6.7)$ \\
T1-weighted sequence & \\
$\quad$ hypointense & $26(86.7)$ \\
isointense & $3(10.0)$ \\
$\quad$ hyperintense & $1(3.3)$ \\
T2-weighted sequence & \\
hypointense & $1(3.3)$ \\
isointense & $0(0.0)$ \\
$\quad$ hyperintense & $29(96.7)$ \\
cystic changes & $5(16.7)$ \\
\hline
\end{tabular}

Operative data were available in 28 patients, in all of whom we achieved gross-total resection of the tumor. In addition, we performed complete resection of the amygdala in 25 patients (89.3\%), partial resection of the hippocampus in $20(71.4 \%)$, and complete resection of the hippocampus in $3(10.7 \%)$. The extent of resection was confirmed by postoperative MR imaging in all but 5 patients. Figure 2 shows typical pre- and postoperative MR images obtained in a patient who underwent resection of a ganglioglioma.

In most cases of dominant temporal lobe tumors, intraoperative or subdural cortical stimulation mapping localized speech function. Since 1995 all procedures (in 24 patients) were performed with image guidance. After initial electrocorticography, a transcortical approach, with or without lateral cortical resection, was used to gain access to the tumor. A gross-total resection was the primary goal of the operation and was aggressively pursued under the microscope while using bipolar cautery and suction prior to 1995 and using ultrasonic aspiration thereafter. Further resection of the amygdala and hippocampus was then performed based on neuropsychological data, Wada testing, pre- and intraoperative testing, and the location of the tumor.

If resection of the amygdala and hippocampus was required, the sequence was as follows. The uncus was resected in a subpial plane under the microscope until the superior landmarks of M1, limen insula, and the endorhinal sulcus were identified. Next, the ventricle was opened by identifying the collateral sulcus and entering

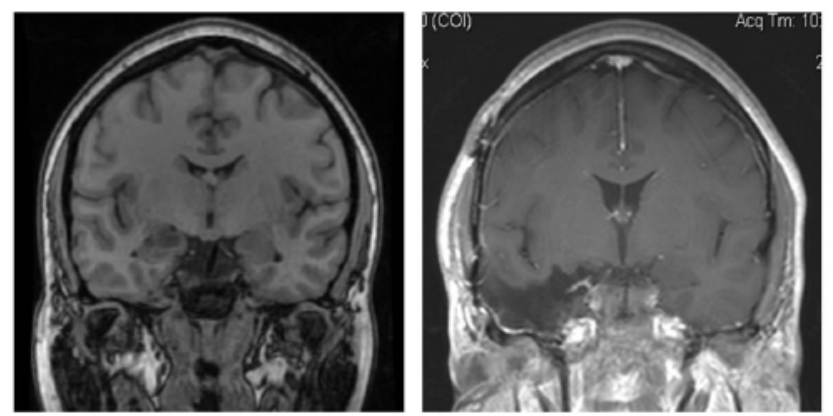

FIG. 2. Preoperative (left) and postoperative (right) MR images acquired after resection of a ganglioglioma. 
Chronic epilepsy with temporal tumors: long-term outcome

TABLE 3: Pathological diagnoses

\begin{tabular}{lc}
\hline Tumor (WHO grade) & No. of Cases (\%) \\
\hline ganglioglioma (I) & $14(36.8)$ \\
DNET (I) & $10(26.3)$ \\
diffuse astrocytoma (II) & $4(10.5)$ \\
pleomorphic xanthoastrocytoma (II) & $3(7.9)$ \\
oligodendroglioma (II) & $3(7.9)$ \\
mixed oligodendroglioma/astrocytoma (II) & $3(7.9)$ \\
astroblastoma (I) & $1(2.6)$ \\
\hline
\end{tabular}

just superior to the collateral eminence. A small retractor elevating the roof of the temporal horn was placed in an axial plane posterior to the visible ventricular prominence of the amygdala at the level of the choroidal point. After identifying the safe axial resection plane for the amygdala at the endorhinal sulcus between M1 and the choroidal point, the thin intralimbic gyrus was subpially aspirated to expose the crural cistern. The amygdala was then resected to the superior level of the endorhinal sulcus. If focal tumor extended above this level, it was resected if it did not surround the anterior choroidal artery or the optic tract. Only rarely do WHO Grade I tumors extend above this line of safe resection. The hippocampus was then removed using ultrasonic aspiration in a subpial plane, exposing and disconnecting hippocampal arteries arising from the posterior cerebral artery. A partial hippocampal resection extended to the level of the choroidal point and a resection of the head and body extended to the ambient cistern, where the tail of the hippocampus recedes medially around the thalamus.

The histological diagnosis was made in accordance with the WHO classification of tumors of the $\mathrm{CNS}^{21}$ (Table 3). Ganglioglioma was the most frequent diagnosis, found in 14 patients (36.8\%), and this was followed by DNET in $10(26.3 \%)$ and diffuse astrocytoma in $4(10.5 \%)$. Of note, on review of the slides by our neuropathologist (E.J.C.), the diagnosis was changed from astrocytoma to ganglioglioma in 3 patients in accordance with the most recent WHO guidelines. ${ }^{21}$ Concomitant ipsilateral mesial temporal sclerosis was noted in 2 patients $(5.3 \%)$, indicative of dual disease.

The mean follow-up duration was 7.7 years (range 1.0-23.1 years). Seizure outcome was classified using Engel's criteria ${ }^{12}$ (Table 4). At the last follow-up, 30 patients (78.9\%) were seizure free, $6(15.8 \%)$ had rare seizures, 2 (5.3\%) exhibited significant improvement, and all patients exhibited a reduction in seizure frequency.

Transient postoperative complications were noted in 7 patients (18.4\%) and included temporary dysphasia in 4 patients, meningitis in 1, hydrocephalus in 1, and pulmonary embolus in 1 . Permanent complications were noted in 1 patient (2.6\%), in whom permanent hemiparesis developed due to intraoperative hemorrhage.

\section{Discussion}

Chronic medically intractable epilepsy secondary to low-grade temporal tumors is a well-recognized entity.
TABLE 4: Seizure outcome based on Engel classification

\begin{tabular}{cc}
\hline Engel Class & No. of Patients (\%) \\
\hline I & $30(78.9)$ \\
II & $6(15.8)$ \\
III & $2(5.3)$ \\
IV & $0(0.0)$ \\
\hline
\end{tabular}

The incidence of low-grade tumors responsible for seizures has been reported to range from 10 to $50 \%$., 1,6,11,25,27 With continuous improvement in imaging modalities, particularly high-resolution MR imaging, such tumors are detected with increasing frequency before surgery. ${ }^{7}$ In determining the most appropriate treatment for patients with tumoral epilepsy, it is important to understand the pathogenesis and natural history of this disease, as well as to have knowledge of treatment options and surgical outcome. In this report we have presented our experience in 38 cases involving chronic intractable epilepsy associated with temporal lobe tumors, and we have discussed our management and outcomes.

\section{Preoperative Evaluation}

In our series, all patients underwent high-resolution MR imaging, video electroencephalography monitoring, neuropsychological testing, and Wada testing. Interestingly, in 2 of our patients the MR imaging findings were interpreted as normal, and the tumor was discovered subsequently on pathological examination. It remains to be seen whether advent of higher-power MR imaging will lead to discovery of more tumors in patients with "normal" imaging findings. In the remainder of the patients, the great majority exhibited lesions that were hypointense on T1weighted sequences and hyperintense on T2-weighted sequences, with no significant enhancement after Gd administration. We observed cystic changes in only a few patients, and there was no significant correlation between MR imaging signal and tumor histology or outcome. This is consistent with previous reports on MR imaging characteristics of tumors associated with epilepsy. ${ }^{7}$

\section{Surgical Treatment}

Temporal lobe resection has long been established as a safe and effective treatment for refractory temporal lobe epilepsy. ${ }^{26}$ In those patients who harbor temporal lobe tumors, however, there has been considerable controversy regarding most appropriate management, with some advocating lesionectomy only, and other arguing for more extensive resection. . $^{3,6,11,17,19,20,29}$ Several possible mechanisms for epileptogenesis by temporal lobe tumors have been proposed, including gliosis, mass effect on surrounding cortex, changes in cellular morphology, and others. ${ }^{2,5,8,13,19}$ Because it is unclear how much the peritumoral tissue contributes to the initiation and/or propagation of seizures, however, the extent of resection that must be performed is not obvious. In a study specifically addressing this issue, it was found that patients treated with lesionectomy alone had lower seizure-free outcomes than those with more extensive electrophysiologically guided 


\section{Ruban et al.}

resection. ${ }^{17}$ In another study, however, postoperative seizure control was achieved in $94 \%$ of patients after complete lesionectomy regardless of the extent of seizure focus resection. ${ }^{1}$ Thus, this issue remains to be resolved, and the only agreement at this time appears to be that gross-total resection, as long as it can be safely performed, should be the minimum goal of surgery. 1,4,6,18,28 $^{-1}$

In our series, we used intraoperative electrocorticography to guide the extent of resection beyond the lesion. In addition to gross-total removal of the tumor, $89 \%$ of our patients underwent resection of the amygdala and $82 \%$ underwent at least a partial resection of the hippocampus. We find that this approach provides for optimal resection of epileptogenic tissue while minimizing the risk of neuropsychological deficits.

By complete resection of the amygdala, we mean resection to the level of the endorhinal sulcus in an axial plane drawn between M1 and the choroidal point. Comparing pre- and postoperative MR images and using coronal and sagittal plane anatomical atlases and cadaveric specimens, it seems likely that what we, and most centers, call a complete resection of the amygdala is really an $80-90 \%$ resection due to technical limitations of the chosen approach. The main nuclei of the amygdala that have dense interconnections with the hippocampus and the temporal neocortex (basal, accessory basal, and lateral nuclei) would be included in this resection. The phylogenetically primitive corticomedial nuclear group reside posteriorly and dorsomedially at the level of the semilunar gyrus. This nuclear group becomes indistinguishable with the basal ganglia and is very close to the optic tract, and thus, it is usually not resected. Because this group is largely a relay nucleus of the lateral olfactory tract, it is thought (although not proved) that it is not critical to resect this relatively small nuclear group.

The extent of resection of the hippocampus was determined by intraoperative landmarks, followed by postoperative imaging. In our experience of $>400$ amygdalohippocampectomies, the definition of partial hippocampal resection was a resection of the head of the hippocampus to the level of the choroidal point where the head transitions to the body. This corresponds to $\sim 2-2.5 \mathrm{~cm}$ of the hippocampus. Complete resection was considered resection of $\sim 5-5.5 \mathrm{~cm}$ of the head and body to the level of the colliculus where the ventricular entry into the occipital horn becomes visible and the tail bends medially behind the thalamus. We did not attempt to resect the tail of the hippocampus.

\section{Pathological Diagnosis}

It has been previously reported that low-grade gliomas account for a great majority of tumors among patients with chronic epilepsy. ${ }^{3,5,15}$ Among the published series, however, there is no consensus regarding which histological entity is the most common. In our series, ganglioglioma was the most common diagnosis, consistent with another report of 216 cases ${ }^{27}$ Other groups have reported astrocytoma ${ }^{11,15,28}$ and $\mathrm{DNET}^{10,19}$ to be the most common. One important note is that on review of the older cases by our neuropathologist, the diagnosis in 3 cases was changed from astrocytoma to ganglioglioma, in ac- cordance with the most recent WHO guidelines, ${ }^{21}$ which may account for the discrepancy with some series. In 2 of our patients, mesial temporal sclerosis was noted in addition to neoplasm, effectively indicating dual disease. This entity has been previously described, and, in accordance with previous recommendations, these patients underwent resection of both the neoplasm and mesial temporal structures. ${ }^{9,14,24}$

\section{Surgical Outcome}

The patients in our series experienced significantly improved outcome with respect to seizure frequency, with status in $78.9 \%$ improving to Engel Class I and in $15.8 \%$ to Engel Class II. These results are in line with other similar series, in which $>80 \%$ of patients were noted to be free of disabling seizures after surgery. ${ }^{6,15,16,19,22,23,28}$ There were no deaths in our series after a mean follow-up of 7.7 years (and as long as 23 years in 1 patient), which again underscores the low malignancy potential of the neoplasms found in these patients.

\section{Conclusions}

Low-grade tumors in the temporal lobe causing chronic medically intractable epilepsy are a common and well-recognized entity. Based on existing data, the most appropriate management appears to be gross-total resection of these tumors, which can be done safely in the majority of cases. Our long-term follow-up suggests that, with complete resection of the tumor and peritumoral epileptogenic tissue, $>90 \%$ of these patients can be expected to be seizure free or have only occasional seizures. Further study is needed to determine the optimal extent of resection and whether it can be limited to the lesion alone or should include peritumoral tissue and mesial temporal structures.

\section{Disclaimer}

The authors report no conflict of interest concerning the material or methods used in this study or the findings specified in this paper.

\section{References}

1. Awad IA, Rosenfeld J, Ahl J, Hahn JF, Luders H: Intractable epilepsy and structural lesions of the brain: mapping, resection strategies, and seizure outcome. Epilepsia 32:179-186, 1991

2. Beaumont A, Whittle IR: The pathogenesis of tumour associated epilepsy. Acta Neurochir (Wien) 142:1-15, 2000

3. Berger MS, Ghatan S, Haglund MM, Dobbins J, Ojemann GA: Low-grade gliomas associated with intractable epilepsy: seizure outcome utilizing electrocorticography during tumor resection. J Neurosurg 79:62-69, 1993

4. Blume WT, Girvin JP, Kaufmann JC: Childhood brain tumors presenting as chronic uncontrolled focal seizure disorders. Ann Neurol 12:538-541, 1982

5. Boon PA, Williamson PD, Fried I, Spencer DD, Novelly RA, Spencer SS, et al: Intracranial, intraaxial, space-occupying lesions in patients with intractable partial seizures: an anatomoclinical, neurophysiological, and surgical correlation. Epilepsia 32:467-476, 1991

6. Britton JW, Cascino GD, Sharbrough FW, Kelly PJ: Low- 


\section{Chronic epilepsy with temporal tumors: long-term outcome}

grade glial neoplasms and intractable partial epilepsy: efficacy of surgical treatment. Epilepsia 35:1130-1135, 1994

7. Bronen RA, Fulbright RK, Spencer DD, Spencer SS, Kim JH, Lange RC: MR characteristics of neoplasms and vascular malformations associated with epilepsy. Magn Reson Imaging 13:1153-1162, 1995

8. Cascino GD: Epilepsy and brain tumors: implications for treatment. Epilepsia 31 (3 Suppl):S37-S44, 1990

9. Cascino GD, Jack CR Jr, Parisi JE, Sharbrough FW, Schreiber $\mathrm{CP}$, Kelly PJ, et al: Operative strategy in patients with MRIidentified dual pathology and temporal lobe epilepsy. Epilepsy Res 14:175-182, 1993

10. Cataltepe O, Turanli G, Yalnizoglu D, Topcu M, Akalan N: Surgical management of temporal tumor-related epilepsy in children. J Neurosurg 102 (3 Suppl):280-287, 2005

11. Drake J, Hoffman HJ, Kobayashi J, Hwang P, Becker LE: Surgical management of children with temporal lobe epilepsy and mass lesions. Neurosurgery 21:792-797, 1987

12. Engel J Jr (ed): Surgical Treatment of Epilepsies. New York: Raven Press, 1987, pp 553-571

13. Fried I, Cascino GD: Lesional surgery, in Engel J Jr (ed): Surgical Treatment of the Epilepsies, ed 2. New York: Raven Press, 1993, pp 501-509

14. Fried I, Kim JH, Spencer DD: Hippocampal pathology in patients with intractable seizures and temporal lobe mass. J Neurosurg 76:735-740, 1992

15. Fried I, Kim JH, Spencer DD: Limbic and neocortical gliomas associated with intractable seizures: a distinct clinicopathological group. Neurosurgery 34:815-824, 1994

16. Iannelli A, Guzetta F, Battaglia D, Iuvone L, Di Rocco C: Surgical treatment of temporal tumors associated with epilepsy in children. Pediatr Neurosurg 32:248-254, 2000

17. Jooma R, Yeh HS, Privitera MD, Gartner M: Lesionectomy versus electrophysiologically guided resection for temporal lobe tumors manifesting with complex partial seizures. J Neurosurg 83:231-236, 1995

18. Jorge CL, Nagahashi-Marie SK, Pedreira CC, Rosemberg S, Valério RM, Valente KD, et al: Clinical characteristics and surgical outcome of patients with temporal lobe tumors and epilepsy. Arq Neuropsiquiatr 58:1002-1008, 2000

19. Kirkpatrick PJ, Honavar M, Janota I, Polkey CE: Control of temporal lobe epilepsy following en bloc resection of lowgrade tumors. J Neurosurg 78:19-25, 1993

20. Lombardi D, Marsh R, de Tribolet N: Low grade glioma in in- tractable epilepsy: lesionectomy versus epilepsy surgery. Acta Neurochir Suppl 68:70-74, 1997

21. Louis DN, Ohgaki H, Wiestler OD, Cavenee WK, Burger PC, Jouvet A, et al: The 2007 WHO classification of tumours of the central nervous system. Acta Neuropathol 114:97-109, 2007

22. Luyken C, Blümbke I, Fimmers R, Urbach H, Elger CE, Wiestler OD, et al: The spectrum of long-term epilepsy-associated tumors: long-term seizure and tumor outcome and neurosurgical aspects. Epilepsia 44:822-830, 2003

23. Packer RJ, Sutton LN, Patel KM, Duhaime AC, Schiff S, Weinstein SR, et al: Seizure control following tumor surgery for childhood cortical low-grade gliomas. J Neurosurg 80:998-1003, 1994

24. Prayson RA, Estes ML, Morris HH: Coexistence of neoplasia and cortical dysplasia in patients presenting with seizures. Epilepsia 34:609-615, 1993

25. Spencer DD, Spencer SS, Mattson RH, Williamson PD: Intracerebral masses in patients with intractable partial epilepsy. Neurology 34:432-436, 1984

26. Wiebe S, Blume WT, Girvin JP, Eliasziw M: A randomized, controlled trial of surgery for temporal lobe epilepsy. N Engl J Med 345:311-318, 2001

27. Wolf HK, Campos MG, Zentner J, Hufnagel A, Schramm J, Elger CE, et al: Surgical pathology of temporal lobe epilepsy. Experience with 216 cases. J Neuropathol Exp Neurol 52:499-506, 1993

28. Zaatreh MM, Firlik KS, Spencer DD, Spencer SS: Temporal lobe tumoral epilepsy: characteristics and predictors of surgical outcome. Neurology 61:636-641, 2003

29. Zentner J, Hufnagel A, Wolf HK, Ostertun B, Behrens E, Campos MG, et al: Surgical treatment of neoplasms associated with medically intractable epilepsy. Neurosurgery 41:378-387, 1997

Manuscript submitted April 9, 2009.

Accepted May 18, 2009.

Portions of this paper were presented in abstract form at the 2008 American Association of Neurological Surgeons Annual Meeting, Chicago, Illinois.

Address correspondence to: Dmitry Ruban, M.D., 1725 West Harrison Street, Suite 1115, Chicago, Illinois 60612. email: dsruban@yahoo.com. 